\title{
Experiencias de conocimientos adquiridos por medio del currículo oculto por profesionales que han egresado de la Carrera de Psicología en Tepatitlán, México
}

\section{Experiences of knowledge acquired through the hidden curriculum by professionals graduated from the School of Psychology in Tepatitlan, Mexico}

\author{
Cándido González Pérez ${ }^{1}$ \\ Universidad de Guadalajara \\ Guadalajara, México \\ candido195913@yahoo.com.mx
}

\author{
Juan Carlos Plascencia de la Torre ${ }^{2}$ \\ Universidad de Guadalajara \\ Guadalajara, México \\ jcplascencia88@hotmail.com
}

Recibido: 12 noviembre 2015 Aceptado: 16 junio 2016 Corregido: 22 julio 2016

Resumen: Los conocimientos de una profesión se obtienen en la universidad por medio de dos vías: a través de los planteamientos académicos vertidos en los programas de estudio, y por medio de habilidades y destrezas particulares que ha adquirido el personal docente y que son transmitidos de manera informal. Se le denomina currículo oculto a todos aquellos conocimientos de importancia que el profesorado enseña voluntaria o involuntariamente de manera extracurricular a sus estudiantes. El objetivo principal de este trabajo es describir, cómo es que los grupos de estudiantes adquirieron saberes y habilidades en su trabajo a partir de conocimientos transmitidos por una parte de sus docentes y con la característica de que esos saberes no formaban parte de los requisitos curriculares. La luz que ofrecen estos resultados proviene de haber realizado entrevistas abiertas a la totalidad de los sujetos egresados de la cohorte que culminó sus estudios en el 2014 en el Centro Universitario de Los Altos de la Universidad de Guadalajara en México. Concretamente, pudimos conocer que adquirieron habilidades que utilizan en su trabajo con respecto al campo neurológico aun sin haber utilizado el material psicométrico que se supone deberían haber usado en las prácticas de laboratorio que no realizaron por la insuficiencia de los componentes necesarios. Hechos similares se observaron con respecto a la aplicación de dinámicas de grupo, en competencias laborales, en elaboración de psicodiagnósticos y en neuropsicología. Todo ello fue posible porque el profesorado que estuvo a cargo de asignaturas diversas, pudo transmitir habilidades que han cultivado a través de la práctica diaria de su profesión y con la característica principal de que esos saberes inculcados no están explícitos en los programas de estudio de la carrera de psicología.

Palabras clave: Egresados y egresadas, currículo oculto, currículo vivo, trabajo profesional.

1 Profesor investigador de la Universidad de Guadalajara en el Centro Universitario de Los Altos, miembro del Sistema Nacional de Investigadores.

2 Auxiliar de investigación, estudiante de la Carrera de Psicología de la Universidad de Guadalajara en el Centro Universitario de Los Altos. 


\begin{abstract}
A profession's knowledge is obtained in college in two ways: through academic approaches poured into the curriculum, and through abilities and skills they have acquired and are transmitted informally by the teachers. It is called hidden curriculum to all the knowledge of importance that teachers teach voluntarily or involuntarily. The main objective of this paper is to describe in particular how the students acquired knowledge and skills in their work from the knowledge transmitted by some of their teachers and with the characteristic that this knowledge is not part of the curriculum requirements. These results come from interviews made to all graduates of the generation 2014 who completed their studies at the University Center of Los Altos University of Guadalajara in Mexico. We present in a more specific manner how psychologists acquired skills they use in their work related to the neurological field even without using the psychometric material that are supposed to be used in laboratory, practice that was not carried out by the lack of necessary component. Similar facts regarding the application of group dynamics in labor skills, development of psychodiagnosis and neuropsychology were observed. All of this was possible because teachers who were in charge of different subjects, transmitted skills they have cultivated through daily practice of their profession and the main characteristic of those that knowledge are not explicit in the Psychology curricula.
\end{abstract}

Keywords: graduates, hidden curriculum, active curriculum, professional work.

El currículo oculto es una fuente invaluable de generación de conocimientos universitarios. Se ha investigado sobre este tópico durante muchos años, aunque la acuñación del concepto data de finales de los años sesenta (Flores, 2004). Los estudios del área educativa se han preguntado siempre el cómo alguna parte del personal docente transmite mayores conocimientos que otra parte, y de cómo algunos profesores y profesoras logran mayor empatía.

Contar con una buena aceptación es uno de los más importantes requisitos para la enseñanza. Las teorías y los conocimientos específicos plasmados en un programa de estudio son explicados de maneras muy diversas y logran mayor conexión docentes que cuentan con más experiencia en el campo laboral que quienes no se han desarrollado suficientemente. Aunque es el mismo currículo vivo, es decir, el formal (Rangel, 2015), cada docente puede añadir mayores habilidades si es que ha tenido acceso a ellas; esas experiencias que no forman parte del programa, se les denomina currículo oculto, porque si bien representa un agregado de conocimientos, no aparece en ningún documento que sea obligación el exponerlos. El currículo oculto puede ser proveedor de destrezas igual o inclusive mayor que las ofrecidas por los programas de estudio con todo y su formalidad, también se le cataloga como el conjunto de normas y lenguajes que aparecen en el funcionamiento de una institución aun cuando no aparezcan explícitamente (Rojas, 2010).

El origen social de docentes que transmiten conocimientos subrepticios es de gran influencia, quienes han acumulado un capital cultural considerable y que generalmente pertenecen a las clases altas son quienes hacen mayores aportes a los conocimientos extracurriculares. El alumnado que proviene de clases medias tiene mayores ventajas educativas que quienes provienen de clases bajas, porque sus familias tienen ventajas por sus privilegios sociales y culturales; generalmente, son más exitosos académicamente, tienen acceso a clases especiales de cómputo, música y sus habilidades les hacen posible situarse de manera cómoda en el sistema escolar (Margolis, 2001, p. 8).

Con respecto a la metodología utilizada en esta investigación, no nos conformamos con una muestra de nuestro universo de estudio, sino que al elegir una generación completa de 
personas egresadas de la carrera de psicología, realizamos lo que se conoce como estudio en caso y los resultados que presentamos, nosotros afirmamos que son válidos para ese grupo en particular, no nos podemos abrogar el hecho de decir que son generales para todos los egresados de la carrera de psicología en las universidades mexicanas. Y tiene la gran ventaja de que corresponde a la totalidad del universo de estudio, ya que se buscó entrevistar a la totalidad de la generación del año 2014; algunos no contestaron o no se les localizó, el resto representa nuestro cien por ciento.

Las entrevistas fueron abiertas y muy amplias en su indagación, aunque en este trabajo se circunscriben los resultados a la educación no formal, es decir, a los conocimientos que adquieren el estudiantado al copiar destrezas de sus docentes, quienes asimismo las obtuvieron de experiencias laborales particulares, no necesariamente de conocimientos curriculares. Un hilo que seguimos los autores de este trabajo, es que esos conocimientos adquiridos por los entonces estudiantes de la carrera de psicología, son utilizados en su ámbito laboral, es decir, ya egresados o egresadas de su formación universitaria y ya inscritos e inscritas en el mercado de trabajo. No explicamos cómo es que los grupos de estudiantes hayan conocido aspectos nuevos en su ámbito profesional, sino cómo los utilizan en su práctica cotidiana años después de haberlos adquirido en las aulas.

En particular, el diseño metodológico que aplicamos se puede describir de la siguiente manera: la unidad de análisis está conformada por los conocimientos que transmite el profesorado y que no están contemplados en el llamado currículo vivo que es el formal y que aparece textualmente en los programas de estudio. Dentro de la totalidad de los conocimientos que adquiere el alumnado de psicología, se indagó a través de entrevistas de larga duración, donde expresaron de viva voz cuáles saberes en particular adquirieron por medio de las experiencias de su profesorado y que no formaban parte de los conocimientos que estaban obligados a transmitir.

Las entrevistas se realizaron en distintos lugares, fueron grabadas y posteriormente analizadas para su exposición. El objeto de estudio estuvo conformado por estudiantes, no se incluye información que hayan proporcionado sus docentes, tal vez en un futuro convenga cerrar el círculo e incluir datos proporcionados por los sujetos generadores de los conocimientos provenientes del currículo oculto.

Recopilamos información de la totalidad de estudiantes que aceptaron participar en las encuetas, y de quienes, el 38\% ofreció testimonios de cómo está utilizando los conocimientos provenientes del currículo oculto. El 62\% restante no forma parte del objeto de estudio de este trabajo, porque de sus evidencias no encontramos que haya utilizado conocimientos que provengan del currículo oculto.

Consideramos muy importante señalar que la proporción de estudiantes que demostró haber obtenido conocimientos provenientes del currículo oculto es perceptiblemente alta, ya que 4 de cada 10 (el 38\% mencionado) han vivido esa experiencia. De ninguna manera se podría afirmar que para determinar la influencia de los conocimientos no formales, debería de incidir en más de la mitad de la población que es el objeto de estudio. Basamos nuestra afirmación en que la universidad provee de vías de conocimiento mediante planes de enseñanza y estos se basan en los saberes enunciados exprofeso, entonces, si mediante 
investigaciones exclusivas, como es el caso de lo que ahora exponemos, se puede demostrar que existen conocimientos que no aparecen en los programas de estudio y que forman parte de las experiencias docentes particulares, de las cuales los alumnos y alumnas obtienen el beneficio de adquirir hábitos específicos, discurrimos relevante el exponer que una proporción cercana a la mitad se ha visto beneficiada.

Por otra parte, el proceso de la categorización de la información consistió en extraer los datos específicos que revelan la aplicación de saberes particulares en el mercado laboral de profesionales de la psicología. Se fueron depurando una vez que se contrastaba su acción con el conocimiento del que existe el compromiso institucional de transmitirse. Las categorías utilizadas son principalmente dos: los saberes que se utilizan en el mercado laboral y que están justificados en los programas de estudio y, por la otra parte, los conocimientos que no están formalizados. En este trabajo, hemos centrado el análisis en la descripción particular de las actividades que los grupos profesionales realizan en su medio laboral y cuya fuente no proviene del currículo formal.

\section{Características del currículo oculto}

El currículum oculto es una especie de fuente de "educación silenciosa" porque en ocasiones se transmite por conductas y ritos que no se explican necesariamente en las lecciones formales. Son modelos de conducta que el personal académico transmite a veces sin proponérselo. Los profesores y profesoras, en el momento de exponer sus experiencias en el campo laboral, predican con el ejemplo; no es necesario sugerir que deben realizar determinadas acciones, el alumnado es receptivo y capta los modelos y patrones que le gustaría adoptar. Un profesor puede dar una cátedra de ética al estar explicando un tema que no sea necesariamente el de los códigos de ética; cuando expone sus experiencias particulares, sus estudiantes aprueban ciertos códigos y los internalizan; cuando se enfrenten a una situación como la aludida, van a actuar de la misma manera como se les explicó. No es necesaria la exposición detallada de las normas escritas sobre la materia. Por ejemplo, cuando una docente expone varios casos de pacientes con tendencias suicidas, no es indispensable conocer reglamentos específicos sobre la materia, lo que aprenden, en este caso, es cuáles fueron los pasos que siguió, en qué pudo tener fracasos y cuáles fueron sus aciertos. Las tendencias suicidas son personales y al mismo tiempo sociales, y cómo actuó la profesional es la enseñanza particular que ofrece en este caso el currículo oculto. La fuente principal del currículo oculto tiene más características de arte que de ciencia, porque en la mayoría de los casos se circunscribe a habilidades aplicadas en forma personal; es decir, no se pueden estandarizar.

Hay una serie de características que aparecen inmersas en el currículum oculto. Entre ellas podemos destacar las siguientes: es omnipresente porque actúa en todos los momentos; es omnímodo porque reviste múltiples formas de influencia; es reiterativo, como lo son las actividades que se repiten de manera casi mecánica en una práctica institucional que tiene carácter rutinario; es invaluable, lo cual provoca que no se valoren en múltiples ocasiones las repercusiones que acarrea, (Carrillo, 2009, p. 2) 
El actuar profesional en cada persona se expresa en el intercambio cara a cara, en el quehacer de la vida cotidiana del psicólogo o psicóloga, en lo singular de cada persona. Las actitudes particulares no se pueden formalizar en el programa de estudio, por eso es oculto, porque no aparece en ningún canon.

El origen social de los padres y madres es un factor de gran relevancia que no debe soslayarse. En un estudio realizado en Estados Unidos, se demostró, a través de una investigación realizada en cinco escuelas primarias de las cuales sus estudiantes provenían de familias con estatus social muy diferenciado, que quienes poseían mayor capital cultural (también económico) tenían más fácil acceso al conocimiento, porque los maestros y maestras utilizaban técnicas y herramientas más sofisticadas. Por ejemplo, de acuerdo con Anyon (1980), en una clase de historia, se utilizaba una película, editada por un padre, alusiva a los temas, lo cual representa un plus para el grupo, pues se les facilita el conocimiento; con esta simple diferencia, estudiantes de la escuela de hijos de madres o padres exitosos en el trabajo, se nutrieron de un currículo oculto al que los otros grupos no tuvieron acceso.

El papel principal de las enseñanzas del currículo oculto, de acuerdo con la teoría, es mantener el statu quo, el transmitir conocimientos relevantes que permitan reproducir a la sociedad o, por lo menos, al espacio particular de la profesión de que se trate (Carrillo, 2009; Margolis, 2001). Si su papel principal es la reproducción, su ámbito de influencia es la cultura, las ideas religiosas e inclusive los prejuicios. En el ámbito profesional de la psicología, si el personal docente es profundamente religioso, transmite sentimientos acordes con sus pensamientos aun cuando, en algunos casos, sea de manera involuntaria. En cuanto a la enseñanza universitaria, la laicidad debe estar asegurada; no obstante, no es difícil encontrar docentes que expresan sus sentimientos, influidos de religión, sin cortapisas.

En un ámbito como el de la sociedad alteña, que es donde se ubica la ciudad de Tepatitlán, existe, según la última información censal, un 98\% de personas católicas, por lo que es poco menos que imposible escuchar que docentes se permitan expresar sus opiniones y que una cantidad considerablemente alta de estudiantes las acepten. El ingrediente religioso es uno de los más importantes en la formación profesional porque está omnipresente. Una característica más del currículo oculto es que los conocimientos, costumbres y los prejuicios se adquirieron en ámbitos no necesariamente universitarios; el personal docente los transmite en la universidad, pero no necesariamente ahí los adquirieron (Muñoz García, 2016).

En virtud de que es imposible homologar el conocimiento porque cada persona vive y adquiere costumbres de manera diferente, el currículo operacional, según Torres y Fernández (2015), ofrece conocimientos universales y el oculto los internaliza de diferentes maneras en cada sujeto receptor. La posición social de cada individuo juega un papel primordial en la transmisión de los conocimientos en particular. En un artículo científico proveniente del ámbito escolar estadounidense, se afirma que es más eficiente la transmisión de conocimientos a las mujeres blancas provenientes de la clase media, si cuentan con una docente blanca proveniente de la clase media (Flores, 2004).

La discriminación es otra fuente de enseñanza del currículo oculto. No son muchas las personas académicas que saben que el currículo oculto cuenta con la otra cara de la moneda. La discriminación como prejuicio es una fuente importante de extensión del "conocimiento", 
los malos ejemplos también son ejemplos. Si el personal docente transmite valores, también transmite antivalores. Es importante destacar un hecho que es fuente de adquisición de conocimientos del currículo oculto: en México la gran mayoría del profesorado universitario es profesionista con alguna especialización y, en segundo lugar, docente (Flores, 2004). Gran parte de las habilidades, destrezas y antivalores se adquirieren fuera del ámbito universitario y luego son transmitidos en los recintos del conocimiento. Es imposible escuchar entre estudiantes de Los Altos decir que "les tocó un compañero que está prietito, pero que es buena gente". También es frecuente observar cómo se desarrollan actividades que están encaminadas a realizarse por un sexo determinado y se vería con malos ojos que los integrantes del otro género las quisieran realizar (Bonnafé y Vidal, 2012). Esto también es currículo oculto. La universidad es no solo el trabajo en el aula, el currículo oculto se transmite en los corredores, los sanitarios (estudiantes que arrojan papeles y estudiantes que limpian el espacio del lavabo utilizado), la cafetería y los jardines (Hafferty, 1998).

El concepto de habitus de Pierre Bourdieu es, de acuerdo con Margolis (2001), en esencia de la Carrera de Medicina Veterinaria y Zootecnia, que deben vestir calzando botas, camisa a cuadros, pantalón de mezclilla y chaleco. Existen actividades donde a las alumnas se les relega. Esta carrera hace un par de decenios era masculina y a las pocas mujeres que se inscribían se les podía señalar de homosexuales por preferir trabajos "propios" de hombres. Currículo oculto son todas las actividades que repiten profesores y alumnos, son las reglas no escritas que prevalecen en toda institución. La manera de expresar el habitus de los alumnos y docentes eran mujeres a las que les gustan las mujeres.

Consideramos que el concepto habitus es primordial cuando hablamos de currículo oculto, porque nos estamos refiriendo a todas aquellas actividades que hace el personal docente y que no se restringen a su actividad frente a grupo en el sentido de transmitir los conocimientos que dicen los libros, sino todas aquellas expresiones de la vida cotidiana: un formulario escrito de manera exprofeso. El habitus se conforma como una de las que los han moldeado en su ámbito profesional. Por ejemplo, un académico que ha tratado y atendido a jóvenes delincuentes ha adquirido una forma especial de comunicarse con ellos, eso no aparece en ningún recetario; una profesional que ha atendido como experta a personas con tendencias suicidas adquiere saberes que obtuvo sin haber aprendido principales categorías para entender lo que significa aprender cosas nuevas, sin que hayan sido escritas ad-hoc, es decir, para entender lo que significa el currículo oculto.

Una última característica palpable del currículo oculto es que se utiliza "violencia simbólica", este concepto es también del cuño del filósofo francés Pierre Bourdieu y representa la otra cara de la moneda del currículo oculto, a saber: lo que está prohibido sin que exista una regla oficial. En concreto, hay dos formas de enseñar o prohibir y se hacen mediante el ejemplo o el amago: se transmiten habilidades por medio de la persona profesional y se obstaculiza hacer algo por medio del uso del lenguaje (escrito o no escrito). La "violencia simbólica" es una forma de imponer patrones sin que exista una norma que lo prescriba (Rojas, 2010, p. 89). Ejemplos: mujeres no pueden llegar a la universidad montando una motocicleta (serían homosexuales por ese solo hecho); los estudiantes de contaduría pública no deben vestir como los de medicina veterinaria (parecerían rancheros); la puntualidad en clase forma parte de la evaluación escolar, aun cuando es una simple norma de rutina la 
convierten en rubro evaluable; la utilización del lenguaje oficial en cada una de las carreras por parte de sus docentes; solo una proporción del alumnado tiene la capacidad de utilizar vehículos de lujo para asistir a clases; el vestir marcas específicas de ropa es exclusividad de un grupo reducido; las acusaciones de homosexualidad en las muy variadas manifestaciones (el albur en un medio de transmisión por excelencia de ideas discriminatorias). Inclusive, las evaluaciones escolares son reflejo de violencia simbólica porque una de las vías para obtener la titulación automática es la "graduación por excelencia" que consiste en haber obtenido un alto promedio escolar. Al estudiantado de psicología les dicen "loqueros" los de otras carreras, o también les endosan "si no pueden con sus problemas, ¿cómo van a poder con los de otros?", se corren los chistes sobre la necesidad de acudir a un psicólogo cuando se tienen problemas matrimoniales "deberías de pedir cita con la psicóloga Violeta, es muy buena en estos asuntos, aunque ya está divorciada", o el caso de que "ve a que te atienda el psicólogo Fernando por tus problemas de alcoholismo, él ya está saliendo de ese problema".

La violencia simbólica establece y reproduce jerarquías. Cabe mencionar que los resultados de la investigación que aquí presentamos explican algunos logros del conocimiento adquirido por las destrezas especiales del profesorado y no exponemos casos de la violencia simbólica, únicamente dejamos constancia de su existencia.

\section{Características de la influencia del currículo oculto de profesionales de la psicología en Tepatitlán}

Para el caso de la realización de este estudio, consideramos únicamente el currículo oculto que se obtiene de forma positiva, es decir, exponemos todos aquellos conocimientos y destrezas obtenidos por el alumnado a través de la experiencia de sus docentes por las actividades que no están observadas en el currículo vivo. Aun cuando están presentes y fácilmente observables las acciones que generan la violencia simbólica, el habitus, y todo lo relacionado con la discriminación, no forma parte del objeto de estudio de esta investigación.

A continuación, se describen las experiencias de 9 profesionales de la piscología que egresaron del Centro Universitario de Los Altos. La cantidad de testimonios de las personas que afirman haber obtenido conocimientos o habilidades provenientes del currículo oculto, representa al 38\% del total, proporción considerablemente alta y que, por esa razón, hemos decidido publicar sus resultados, por cuanto el fenómeno es de gran relevancia y puede servir de punto de comparación con otros trabajos que se hayan propuesto sobre el mismo género. Cada uno de los siguientes nueve párrafos corresponde a cada uno de los egresados mencionados.

La utilización del material psicométrico no era suficiente y se organizaba al alumnado para turnárselo. Los saberes en el área del campo clínico y neurológico le proporcionaron conocimientos extras que ha utilizado en su campo de acción porque es voluntaria en el área clínica en un instituto del servicio público. Aprendió mucho en las prácticas en especial en la "escucha activa". Una actividad especial que desempeña es sobre un curso para la prevención del suicidio con adolescentes. Aplica test a medio centenar de jóvenes y tiene tres pacientes.

En la enseñanza de dinámica de grupos adquirió destrezas especiales para entrar en contacto con grupos, la correcta utilización del lenguaje, el manejo de un léxico más amplio, 
facilidad de palabra, análisis del discurso y análisis de los símbolos y su interpretación. Eso lo obtuvo con mayor práctica, no lo proporciona el programa formal de estudios. En particular, trabaja con grupos de salud, ofrece charlas psicoeducativas para prevenir enfermedades.

Obtuvo experiencia en las asignaturas de especialidad en el tema laboral, aunque son preferentemente teóricas. Ella adquirió conocimientos que la llevaron a preparar conferencias, porque se desempeñó como auxiliar de una investigadora en el área de las ciencias sociales. En particular, afirma contar con habilidades específicas para la gestión, la organización profesional y la intervención pedagógica.

De la asignatura de psicología clínica obtuvo destrezas especiales para la utilización de varios enfoques de psicoterapia y tiene habilidades para realizar psicodiagnósticos. Encontró utilidad práctica a sus conocimientos, porque está trabajando como persona especializada en la prevención de delitos por medio de programas de inteligencia emocional, prevención de la violencia y las adicciones.

Se organizaba al estudiantado originario de un mismo municipio y quien fuera responsable de la utilización de material de psicometrías, lo compartía con sus colegas. Las asignaturas de las que obtuvo conocimientos especiales fueron las del bloque metodológico y la de corrientes en psicología. Tiene habilidades para la investigación y aun cuando la carrera es propicia para ello, es poca la cantidad de estudiantes que se inclina por esta actividad profesional. Considera haber adquirido habilidades específicas en las actividades verbales y en el manejo de grupos a través de los conocimientos extracurriculares que aportaron sus docentes del bloque metodológico.

La clase de neuropsicología la marcó en su formación porque adquirió conocimientos por encima del promedio de sus pares. La asignatura aun y cuando no es de las más populares, le definió su rumbó profesional y desde entonces hasta la fecha, se ha propuesto realizar un posgrado en esa área para luego desempeñarse en un hospital. En particular, afirma que las competencias particulares que adquirió cuando cursaron esa materia, fueron el diagnóstico, la observación y el análisis.

A esta alumna la dejó marcada la materia de técnicas de psicodiagnóstico Infantil y de adultos. La favoreció en "afinar su ojo clínico" y decidir su tendencia laboral hacia la terapia y las entrevistas. La planeación y la buena estructuración de las organizaciones fue un plus que recibió en su formación y de lo cual los programas de estudio ofrecen poco. Ella lo aprendió de su maestro en esta asignatura, razón por la cual, aun cuando no cuenta con un empleo remunerado, se desempeña como voluntaria en una institución pública donde aplica esos conocimientos que consisten en elaborar psicoterapias y valoración psicológica.

$\mathrm{Al}$ siguiente profesional le gustaría especializarse en el área de psicoterapia, dice que "a nivel personal es allí donde encontré más satisfacción y puse en práctica mis habilidades y encontré la posibilidad de cumplir con esa meta en mi vida personal, que es poder servir a la sociedad donde interactúo, suena un poco romántico, pero a nivel personal es lo que quiero. Para esto, creo que las competencias que ahí adquirí fueron la evaluación, psicometrías, entrevistas, observación, aplicación de técnicas y dinámicas terapéuticas, análisis del discurso y una formación muy humana.

Las prácticas profesionales les ofrecieron un plus a sus conocimientos, ahí aprendió y puso en práctica todo lo relacionado con la psicología. Se inclinó por la psicoterapia con un 
enfoque sistémico y utilizó sus habilidades en las prácticas clínicas. Dice que "para ejercer esta área, creo que cuento con las competencias de contacto con las personas como niños y adultos. Manejo mucho la empatía”.

\section{Conclusiones}

El currículo oculto que influyó de manera positiva en la formación de profesionistas que egresan del Centro Universitario de Los Altos y de quienes se observa en la práctica cotidiana, en los espacios laborales en que se desempeñan, adquisiciones directamente de los profesores y profesoras que les transmitieron sus habilidades y, muy en especial, de quienes han logrado éxito en sus prácticas profesionales. La peculiaridad que resaltamos a partir de la investigación es que esos conocimientos no están explícitos en los programas de estudio, sino son fruto exclusivo de las experiencias adquiridas en el trabajo cotidiano de los especialistas. El plan de estudios en que se basa el personal docente para transmitir los saberes de la psicología es revisado y actualizado constantemente; no obstante, siempre hay erudiciones que, aunque se transmiten, no están documentadas en los programas escolares; esa es la particularidad del currículo oculto, que son saberes transmitidos y no fundamentados en documentos.

Los sujetos egresados expresaron sus experiencias particulares y afirmaron que dos espacios particulares donde fueron receptáculos de diversos saberes, los conforman las prácticas profesionales y el servicio social. Las prácticas profesionales son actividades que realizan de manera profesional en algún espacio público al que se asignan formalmente por parte de la universidad y que generalmente son espacios de atención a la salud. El servicio social representa un contrato de trabajo sin remuneración económica que deben cumplir a manera de retribuir algo a la sociedad que pagó impuestos para que tuvieran acceso gratuito a la educación superior en México.

Una proporción considerablemente alta del grupo entrevistado, el 38\% del total, afirma con testimonios contundentes el haber adquirido conocimientos y destrezas por parte de docentes que atendieron su formación universitaria y que esas sapiencias las han reproducido en su ambiente laboral.

Se cubrió con creces el objetivo de haber realizado la investigación de la que aquí se presentan los resultados, porque los grupos egresados describieron puntualmente cuáles fueron las habilidades y destrezas que les transmitieron sus profesores y profesoras, aun cuando no formaban parte de lo expresado en los programas de estudio. De esa manera seis de las personas entrevistadas han logrado fortalecer sus competencias profesionales en el área de la psicología clínica, ejerciendo como psicoterapeutas, aplicando pruebas psicométricas y evaluando en el área de la neuropsicología. Por otra parte, dos lograron permanecer como expositora y expositor psicoeducativos, ofreciendo charlas de programas de prevención de suicidios y promoción de la salud en organizaciones del ámbito de la salud; y finalmente, un alumno especializado en el área de la investigación científica refiere haber adquirido nuevas competencias a partir de laborar como auxiliar de investigación, experiencia que no estaba planeada dentro de su formación curricular. Hoy en día ejerce en el área de la intervención pedagógica.

Como se aprecia, el currículo oculto se hace presente en los sujetos egresados, sin provenir de los programas de estudios de la carrera, sino de una experiencia vivencial que 
no está plasmada en el currículo vivo. Es a partir de esto que se le denomina la educación silenciosa, ya que nace desde lecciones informales y actos más vivenciales. En la práctica, adquiere dos características: una positiva y otra negativa. A la positiva se le puede asociar con los conocimientos que se adquieren a partir de la experiencia particular de cada docente de la universidad, lo que en términos académicos se conoce como habitus; la negativa es reconocida como "violencia simbólica". En los casos que se mencionan en este trabajo se hace énfasis en particular a la proveniente del habitus y la segunda se deja apenas señalada.

\section{Referencias}

Anyon, J. (1980). Social class and the hidden curriculum of work [La clase social y el currículum oculto de la obra]. Journal of education, 162(1) 67-92. Recuperado de http:/l www.jeananyon.org/docs/anyon-1980.pdf

Bonnafé, J., y Vidal Otero, C. (enero-febrero, 2012). El poder del currículo oculto en la educación básica en México: Alternativas para su evolución. Revista Internacional por la Psicología y Educación Integral, 1(2), 4-20.

Carrillo Siles, B. (2009). Importancia del currículum oculto en el proceso de enseñanzaaprendizaje. Innovación y experiencias educativas, 1-10. Recuperado de http://www.csi-csif. es/andalucia/modules/mod ense/revista/pdf/Numero 14/BEATRIZ CARRILLO 2.pdf

Flores García, G. (2004). El currículum oculto y la sobrevivencia en la Universidad. Un ejemplo de la enseñanza en la historia. Cuarto Congreso Nacional y Tercero Internacional: Retos y expectativas de la Universidad. (pp. 1-18). Saltillo, Coahuila: Universidad Autónoma de Coahuila.

Hafferty, F. W. (1998). Beyond curriculum reform: confronting medicine's hidden curriculum [Más allá de la reforma curricular : hacer frente curriculum oculto de la medicina]. Academic Medicine 73 (4), 403-7. doi: http://dx.doi.org/10.1097/00001888-199804000$\underline{00013}$

Margolis, E. (2001). The hidden curriculum in higher education [El curriculum oculto en la educación superior]. New York: Psychology Press.

Muñoz García, T. (2016). Hacia un nuevo diseño curricular el papel de las variables. Campo Abierto. Revista de Eduacación, 7(1), 103-114.

Rangel, H. (2015). Una mirada internacional de la construcción curricular. Por un currículo vivo, democrático y deliberativo. REDIE Revista electrónica de Investigación Educativa, 17(1). Recuperado de http://redie.uabc.mx/redie/article/view/380/992 
Rojas Tudela, F. L. (2010). Curriculum oculto en la educación en derecho. Revista Integra Educativa 3, (3), 87-95.

Torres Chirinos, A., y Fernández Sánchez, E. (2015). Problemas conceptuales del curriculum. Hacia la implementación de la transversalidad curricular. Opción. 31(77), 95-110. Recuperado de http://www.redalyc.org/articulo.oa?id=31041172006 